\title{
DNA methylation of human endogenous retrovirus in systemic lupus erythematosus
}

\author{
Jeerawat Nakkuntod $^{1}$, Pattadon Sukkapan ${ }^{2}$, Yingyos Avihingsanon ${ }^{3}$, Apiwat Mutirangura ${ }^{4}$ \\ and Nattiya Hirankarn ${ }^{2}$
}

Previous studies have reported that T cells from active systemic lupus erythematosus (SLE) patients contained global hypomethylation and demethylation at the promoter of several genes, which may contribute to the pathogenesis of the disease. Currently there are scarce data on methylation of retroelements in patients with SLE. We estimated and compared the methylated levels of human endogenous retroviruses (HERV)-E and HERV-K in normal and SLE CD3 + CD4 + T lymphocytes, CD8 $+\mathrm{T}$ and $\mathrm{B}$ lymphocytes by using combined bisulfite restriction analysis-interspersed repetitive sequences (COBRA-IRS). HERV-E LTR2C methylation level in CD3 + CD4 + T lymphocytes of active SLE was significantly lower than inactive SLE and normal controls $(\boldsymbol{P}=\mathbf{0 . 0 2 3}$ and 0.035 , respectively). Surprisingly, HERV-K LTR5_Hs hypomethylation was significantly detected in CD3 + CD4 + T lymphocytes from patients with inactive SLE when compared with the active SLE and normal controls $(P=0.027$ and 0.002 , respectively). Demethylation of HERV-K LTR5_Hs in B cells was also detected when compared with the normal controls $(P=0.048)$. Furthermore, the hypomethylation of HERV-E LTR2C in CD3 + CD4 + T lymphocytes was positively correlated with lymphopenia in active SLE, whereas the hypomethylation of HERV-K LTR5_Hs was significantly correlated with complement activity and Systemic Lupus Erythematosus Disease Activity Index score. In summary, for each lymphocyte subset in patients with SLE, IRS hypomethylation was found to be type specific. Further studies are needed to confirm and explain these observations.

Journal of Human Genetics (2013) 58, 241-249; doi:10.1038/jhg.2013.6; published online 7 March 2013

Keywords: DNA methylation; HERV; systemic lupus erythematosus

\section{INTRODUCTION}

Systemic lupus erythematosus (SLE) is primarily characterized by the production of autoantibodies against different components of the cell nucleus and double-stranded DNA. These autoantibodies or immune complexes can induce inflammation that can result into glomerulonephritis, arthritis, serositis, vasculitis and neurological disorders or even end-organ damage. The precise etiopathogenesis of SLE is unclear but several studies have suggested that genetic and environmental factors are important for the development of SLE. ${ }^{1}$ Furthermore, there is increasing evidence that epigenetic modifications or changes in the genetic expression that does not change the DNA sequence contribute to the pathogenesis of SLE. ${ }^{2-4}$

DNA methylation occurs after the DNA is synthesized. In eukaryotes, slight modifications of the CpG dinucleotides occur at the fifth cytosine ring. These regions of the genome are also known as the $\mathrm{CpG}$ islands, which are rich in CpG pairs. Most of CpG islands overlap the $5^{\prime}$ end of the gene regions, including the promoter and the first exon sequences. In general, the promoters of active genes and tissue-specific genes are hypomethylated whereas inactive genes are methylated or silenced.
Several independent studies around the world have suggested that the pathogenesis of SLE is associated with DNA globally hypomethylation of CD4 $+\mathrm{T}$ lymphocytes of SLE patient comparing to normal group. ${ }^{5-8}$ In a mice model, it was shown that syngeneic recipient mice would develop lupus-like disease when it received CD4 $+\mathrm{T}$ cells treated with 5-azacytidine or procainamide. ${ }^{9,10}$ Because of this, it has been suggested that hypomethylated $\mathrm{CD} 4+\mathrm{T}$ cells are the main culprits of SLE. Aside from that, it has been shown that some molecules related to disease pathogenesis such as lymphocyte function-associated antigen 1, perforin, CD70, and CD40L were overexpressed in autoreactive $\mathrm{CD} 4+\mathrm{T}$ cell due to promoter hypomethylation. ${ }^{11-14}$ Despite this, the roles and functions of hypomethylated genes continue to elude scientists around the world. In the human genome, excluding CpG-rich sequences within the genes, $\sim 45 \%$ of interspersed repetitive sequences (IRSs) are methylated cytosines. ${ }^{15,16}$ IRSs can be divided into DNA transposons and retroelements, which contribute to about 2.8 and $42.2 \%$ of the human genome, respectively. ${ }^{17}$ Retroelements can further be divided into two groups based on the presence or absence of long terminal repeats (LTRs). Two high copy number of non-LTR retroelement are long

\footnotetext{
${ }^{1}$ Medical Microbiology Interdisciplinary Program, Graduate School Chulalongkorn University, Bangkok, Thailand; ${ }^{2}$ Lupus Research Unit, Department of Microbiology, Faculty of Medicine, Chulalongkorn University, Bangkok, Thailand; ${ }^{3}$ Lupus Research Unit, Department of Medicine, Faculty of Medicine, Chulalongkorn University, Bangkok, Thailand and 4Department of Anatomy, Faculty of Medicine, Center of Excellence in Molecular Genetics of Cancer and Human Diseases, Chulalongkorn University, Bangkok, Thailand Correspondence: Dr N Hirankarn, Lupus Research Unit, Department of Microbiology, Faculty of Medicine, Chulalongkorn University, Rama 4 road, Bangkok 10330, Thailand. E-mail: Nattiya.H@chula.ac.th

Received 19 July 2012; revised 19 January 2013; accepted 22 January 2013; published online 7 March 2013
} 
interspersed nuclear elements (LINEs) (20.1\%) and short interspersed nuclear elements (for example, ALU) (13.1\%). ${ }^{18}$ Approximately $8.2 \%$ of the LRT retroelements are human endogenous retroviruses (HERVs) ${ }^{18}$ Even though IRSs have often been referred to as junk DNA sequence but several studies have suggested that its functional role is to regulate neighboring genes of enhancers, repressors, alternative promoter, a new exon-coding protein and polyadenylation signal. ${ }^{19-21}$ Several studies from various fields have studied the status of IRS methylation. For example, in the field of cancer, hypomethylated LINE-1 is the most commonly studied IRS element. ${ }^{22}$ Hypomethylated LINE-1 has been shown to destabilize and alter the gene. As a result of this finding, hypomethylated IRS can be used as a prognostic marker in detecting cancer. ${ }^{23-25}$ Our previous study has shown that hypomethylated LINE-1, but not Alu, was associated with SLE $^{26}$ whereas hypomethylated Alu and HERV-K were often found in the elderly. ${ }^{27}$ On the basis of these studies, hypomethylation of IRS is also disease specific but the functional consequences of these IRSs hypomethylation are unknown.

Interestingly, peripheral blood mononuclear cells (PBMCs) from SLE and rheumatoid arthritis patients have increased expressions of HERV-E and HERV-K gag genes, respectively. ${ }^{28-30}$ The transcription of HERV-E gag gene is also positively correlated with plasma blood concentrations of anti-U1 ribonucleoprotein and anti-Sm antibodies. $^{29}$ Furthermore, demethylation in HERV-E alternative promoter of CD5 gene in SLE patients has been shown to upregulate CD5-E1B isoform in SLE B cells. ${ }^{31}$ This information supports the theory that IRSs can regulate adjacent cellular genes and contribute to the pathogenesis of SLE. However, the global methylation profile of HERV in SLE patients is still limited.

In this study, a combined bisulfite restriction analysis-HERV (COBRA-HERV) was developed to investigate whether there were any differences in the methylated levels of LTR retrotransposons in $\mathrm{CD} 3+\mathrm{CD} 4+\mathrm{T}$ lymphocytes, $\mathrm{CD} 3+\mathrm{CD} 8+\mathrm{T}$ and $\mathrm{B}$ lymphocytes among patients with SLE and normal healthy controls. Only specific subclass of LTR namely, HERV-E LTR2C and HERV-K LTR5_Hs that have been reported to be associated with autoimmune diseases were used in this study. ${ }^{28-30}$

\section{MATERIALS AND METHODS}

\section{Subjects}

Twenty-three patients with SLE were recruited from the King Chulalongkorn Memorial Hospital (Table 1). Patients who had at least four diagnostic criteria for SLE according to the American College of Rheumatology classification were enrolled into the study. Disease activity was assessed by the Systemic Lupus Erythematosus Disease Activity Index 2000 (SLEDAI-2K). ${ }^{32}$ Inactive disease $(n=7)$ was defined as SLEDAI- $2 \mathrm{~K}$ score of $\leqslant 6$ whereas active disease $(n=16)$ had to have a SLEDAI-2K score of $>6 .{ }^{33}$ Ten healthy female volunteers with no history of any autoimmune disease served as normal controls. The average age of the inactive SLE, active SLE and normal control was $30 \pm 7.1,31.4 \pm 7.7$ and $28.2 \pm 1.9$ years, respectively (mean \pm s.d.). Informed consent was obtained from each patient before entering the study. The trial was approved by the the Institutional Review Board of the Faculty of Medicine, Chulalongkorn University, Bangkok, Thailand.

\section{Cell isolation}

The PBMCs were prepared by Ficoll density gradient centrifugation. B- and T-cell subsets were further isolated from PBMCs by magnetic beads as described. ${ }^{26}$

\section{Cell lines}

Ten carcinoma and lymphoma cell lines were used: cervical (HeLa, SiHa and CaSki) colorectal (RKO), hepatocellular (HepG2), larynx (HEp-2) and
Table 1 Patient demographics and medications

\begin{tabular}{|c|c|c|c|c|c|}
\hline Patient & Group & $\begin{array}{c}\text { Age } \\
\text { (years) }\end{array}$ & Sex & SLEDAI-2K & Medications \\
\hline 1 & Active & 35 & $\mathrm{~F}$ & 12 & $\begin{array}{l}\text { Oral Prednisolone } 20 \mathrm{mg} \text { per day, } \\
\text { Cellcept } 1.5 \mathrm{~g} \text { per day }\end{array}$ \\
\hline 2 & Active & 33 & $\mathrm{~F}$ & 8 & $\begin{array}{l}\text { Oral Prednisolone } 25 \text { mg per day, } \\
\text { Endoxan } 50 \text { mg per day }\end{array}$ \\
\hline 3 & Active & 33 & $\mathrm{~F}$ & 14 & Oral Prednisolone $15 \mathrm{mg}$ per day \\
\hline 4 & Active & 33 & $\mathrm{~F}$ & 10 & Imuran 75 mg per day \\
\hline 5 & Active & 38 & $\mathrm{~F}$ & 17 & Oral Prednisolone $5 \mathrm{mg}$ per day \\
\hline 6 & Active & 30 & $\mathrm{~F}$ & 20 & $\begin{array}{l}\text { Oral Prednisolone } 10 \mathrm{mg} \text { per day, } \\
\text { Cellcept } 1.5 \mathrm{~g} \text { per day }\end{array}$ \\
\hline 7 & Active & 24 & $\mathrm{~F}$ & 20 & Oral Prednisolone $10 \mathrm{mg}$ per day \\
\hline 8 & Active & 25 & $\mathrm{~F}$ & 10 & Oral Prednisolone $10 \mathrm{mg}$ per day \\
\hline 9 & Active & 36 & $\mathrm{~F}$ & 8 & $\begin{array}{l}\text { Oral Prednisolone } 7.5 \mathrm{mg} \text { per day, } \\
\text { Imuran } 50 \text { mg per day }\end{array}$ \\
\hline 10 & Active & 41 & $\mathrm{~F}$ & 9 & Oral Prednisolone $10 \mathrm{mg}$ per day \\
\hline 11 & Active & 37 & M & 12 & $\begin{array}{l}\text { Oral Prednisolone } 15 \mathrm{mg} \text { per day, } \\
\text { Endoxan } 1400 \mathrm{mg} \text { IV } \times 6 \text { monthly }\end{array}$ \\
\hline 12 & Active & 15 & $\mathrm{~F}$ & 16 & Endoxan $1000 \mathrm{mg}$ IV $\times 6$ monthly \\
\hline 13 & Active & 42 & $\mathrm{~F}$ & 16 & Oral Prednisolone $5 \mathrm{mg}$ per day \\
\hline 14 & Active & 20 & $\mathrm{~F}$ & 18 & Oral Prednisolone $15 \mathrm{mg}$ per day \\
\hline 15 & Active & 32 & $\mathrm{~F}$ & 9 & None \\
\hline 16 & Active & 27 & $\mathrm{~F}$ & 16 & None \\
\hline 17 & Inactive & 35 & $\mathrm{~F}$ & 4 & $\begin{array}{l}\text { Oral Prednisolone } 5 \mathrm{mg} \text { per day, } \\
\text { Cellcept } 500 \mathrm{~g} \text { per day }\end{array}$ \\
\hline 18 & Inactive & 30 & $\mathrm{~F}$ & 0 & Oral Prednisolone $10 \mathrm{mg}$ per day \\
\hline 19 & Inactive & 37 & $\mathrm{~F}$ & 0 & Oral Prednisolone $2.5 \mathrm{mg}$ per day \\
\hline 20 & Inactive & 16 & $\mathrm{~F}$ & 6 & Oral Prednisolone $10 \mathrm{mg}$ per day \\
\hline 21 & Inactive & 27 & $\mathrm{~F}$ & 5 & Oral Prednisolone $10 \mathrm{mg}$ per day \\
\hline 22 & Inactive & 27 & $\mathrm{~F}$ & 4 & Oral Prednisolone $10 \mathrm{mg}$ per day \\
\hline 23 & Inactive & 37 & $\mathrm{~F}$ & 0 & None \\
\hline
\end{tabular}

Abbreviations: F, female; IV, intravenous; M, male.

leukemia (K-562, Daudi, Jurkat and MOLT-4). The cells were cultured in Dulbecco's modified Eagle's medium or RPMI1640 (Gibco BRL, Life Technologies, Pairly, UK), supplemented with $10 \%$ heat-inactivated fetal bovine serum (Sigma, St Louis, MO, USA) and incubated at $37^{\circ} \mathrm{C}$ in $5 \% \mathrm{CO}_{2}$.

\section{COBRA validation using calibration DNA}

To test how the COBRA estimation correlate to the defined methylation values, we validate our method using fully methylated and unmethylated human control DNA that was bisulphite treated (EpiTect PCR control DNA; Qiagen, Hilden, Germany). The control DNA was mixed in different ratios to obtain calibration samples with range of methylation percentages of $0,12.5,25,37.5$, $50,62.5,75,87.5$ and $100 \%$, respectively. ${ }^{34}$

\section{Pyrosequencing}

Bisulfited DNA were amplified by using primers with the following sequences: HERV-E forward primer 5'-TTT TGT TAG TTG ATG T(A/G)(G/T) GTA-3', HERV-E reverse biotinylated primer $5^{\prime}$-CCC CAA AAA AAA AAT TC(C/T)T AAC C- $3^{\prime}$ and HERV-K forward primer $5^{\prime}$-ATA TTA AGG GAA TTT AGA GGT TGG-3', HERV-K reverse biotinylated primer $5^{\prime}$-CCC CTA CAC ACC TAT AAA TAT TTC- $3^{\prime}$. Final total PCR volume of $20 \mu \mathrm{l}$ was performed under the following conditions: initial denaturation $95^{\circ} \mathrm{C}$ for $5 \mathrm{~min}$, followed by 35 cycles of $1 \mathrm{~min}$ at $95^{\circ} \mathrm{C}$ and later with either $1 \mathrm{~min}$ at $58^{\circ} \mathrm{C}$ for HERV-E or $60^{\circ} \mathrm{C}$ for HERV-K and $1 \mathrm{~min}$ at $72^{\circ} \mathrm{C}$, and final elongation $7 \mathrm{~min}$ at $72^{\circ} \mathrm{C}$. Biotinylated PCR products were immobilized onto Streptavidin Sepharose HP beads (GE Healthcare, Slough, UK). Biotinylated strands were selected by Vacuum Prep Workstation. Beads were released into plates with $0.4 \mu \mathrm{m}$ sequencing forward primer. The HERV-E and HERV-K methylated levels were quantitated by using the PSQ HS96 Pyrosequencing System. The ratio of C to 
T nucleotides was evaluated for HERV-E and HERV-K methylation. All values were represented by multiplying the methylated peak height by 100 and dividing it by the sum of the methylated peak height and non-methylated peak height.

\section{Combined bisulfite restriction analysis}

DNA was extracted from collected cells by using a salting out method. ${ }^{35}$ Bisulfite conversion of DNA was performed by using the EZ DNA methylation kit (Zymo Research, ZYMO RESEARCH CORP., Orange, CA, USA) according to the manufacturer's instructions. To compare the methylation of global HERV-E and HERV-K, bisulfited DNA was amplified by using primers with the following sequence: HERVE forward $5^{\prime}$-TTTTGTTAGTTGATGTRKGTA-3', HERVE reverse $5^{\prime}$-CCCCAAAAAAAAAATTCYTAACC- $3^{\prime}$ and HERVK forward 5'-ATATTAAGGGAATTTAGAGGTTGG-3', HERVK reverse $5^{\prime}$-CCCC TACACACCTATAAATATTTC- ${ }^{\prime}$. These sequences were selected because it was located at the LTR of HERV-E and HERV-K. With these primers, we were also able to amplify subclasses of HERV-E and HERV-K LTRs known as LTR2C and LTR5_Hs, nomenclatures obtained from the Repbase database. ${ }^{36}$ A total of 77 HERV-E LTR2C and 283 HERV-K LTR5_Hs were amplified after bisulfite treatment by using an in silico PCR of BiSearch. ${ }^{37,38}$ PCR was performed under the following conditions: initial denaturation at $95^{\circ} \mathrm{C}$ for $5 \mathrm{~min}$, followed by 35 cycles of $1 \mathrm{~min}$ at $95^{\circ} \mathrm{C}$ and later with either $1 \mathrm{~min}$ at $58^{\circ} \mathrm{C}$ for HERV-E or $60^{\circ} \mathrm{C}$ for HERV-K and $1 \mathrm{~min}$ at $72^{\circ} \mathrm{C}$, and final elongation $7 \mathrm{~min}$ at $72^{\circ} \mathrm{C}$. The PCR products were digested with 2 units of Tail (Fermentas, Thermo Fisher Scientific Inc., Waltham, MA, USA) in a total final volume of $10 \mu \mathrm{l}$ to detect HERV-E (126bp) and HERV-K (156bp). Each reaction was incubated overnight at $65^{\circ} \mathrm{C}$ and then electrophoresed on an $8 \%$ non-denaturing polyacrylamide gels. The intensities of the DNA fragments were measured on a PhosphoImager by using the ImageQuant software (Molecular Dynamics, GE Healthcare, Slough, UK). HERV-E-positive methylated amplicons yielded 91-, 82-, 73- and 53-bp fragments whereas HERV-K-positive methylated yielded 112- and 44-bp fragments. The methylation level was calculated as a percentage (the intensity of the digested methylated fragment divided by the sum of the undigested and digested amplicons). To reduce inter-assay variability, bisulfite-treated DNA from different cell lines were used as controls for HERV-E (HeLa, Daudi and Jurkat) and HERV-K (K-562, HepG2 and CaSki) assays. For each cell line, the methylated levels were standardized. Once the methylated levels were standardized, the methylated levels for all of the experiments were adjusted according to the controls.

For COBRA for unique HERV-E LTR2C, locus-specific HERV were amplified by using one primer bind at gene-specific sequences and another at LTR2C sequence. Primers for HERV-E LTR2C-APC2 were 5'-TAA AAG TAA GAT AAA TAG ATA A- $3^{\prime}$ and $5^{\prime}$-CCT TTT TAA AAA CTT ACA ACT- $3^{\prime}$. PCR products of LTR2C-APC2 were digested with TaiI (New England Biolabs, Beverly, MA, USA).

\section{Cloning and sequencing}

For bisulfite DNA sequencing of HERV-E LTR2C-APC2, bisulfite-treated DNA was amplify by using primers $5^{\prime}$-AAG TAA GAT AAA TAG ATA ATT TTG G- $3^{\prime}$ and 5'-AAA ACT CCT AAC ACT AAA TTA AAA-3'. The PCR products were cloned into the pGEM-T easy vector (Promega, Santhan, UK). Ten independent clones were sequenced for each of the amplified fragments.

\section{Statistical analyses}

Statistical significance was determined by applying an independent sample $t$-test. Pearson's correlation coefficient was used to examine the relationship between two continuous variables. All analyses were performed by using SPSS, version 11.5 (SPSS Inc., Chicago, IL, USA).

\section{RESULTS}

\section{COBRA-LTRs design and set up}

The primer design for HERV in this study was based on the HERV-E clone 4-1 and HERV-K10 gag transcripts aligned from Genbank with the accession no. M10976 and no.M14123 sequences, respectively. To identify LTR subclasses, HERV sequences were analyzed by Repbase nomenclature. ${ }^{36}$ Subclasses of HERV-E clone 4-1(M10976) and HERV-K10 (M14123) were identified to be LTR2C and LTR5_Hs,
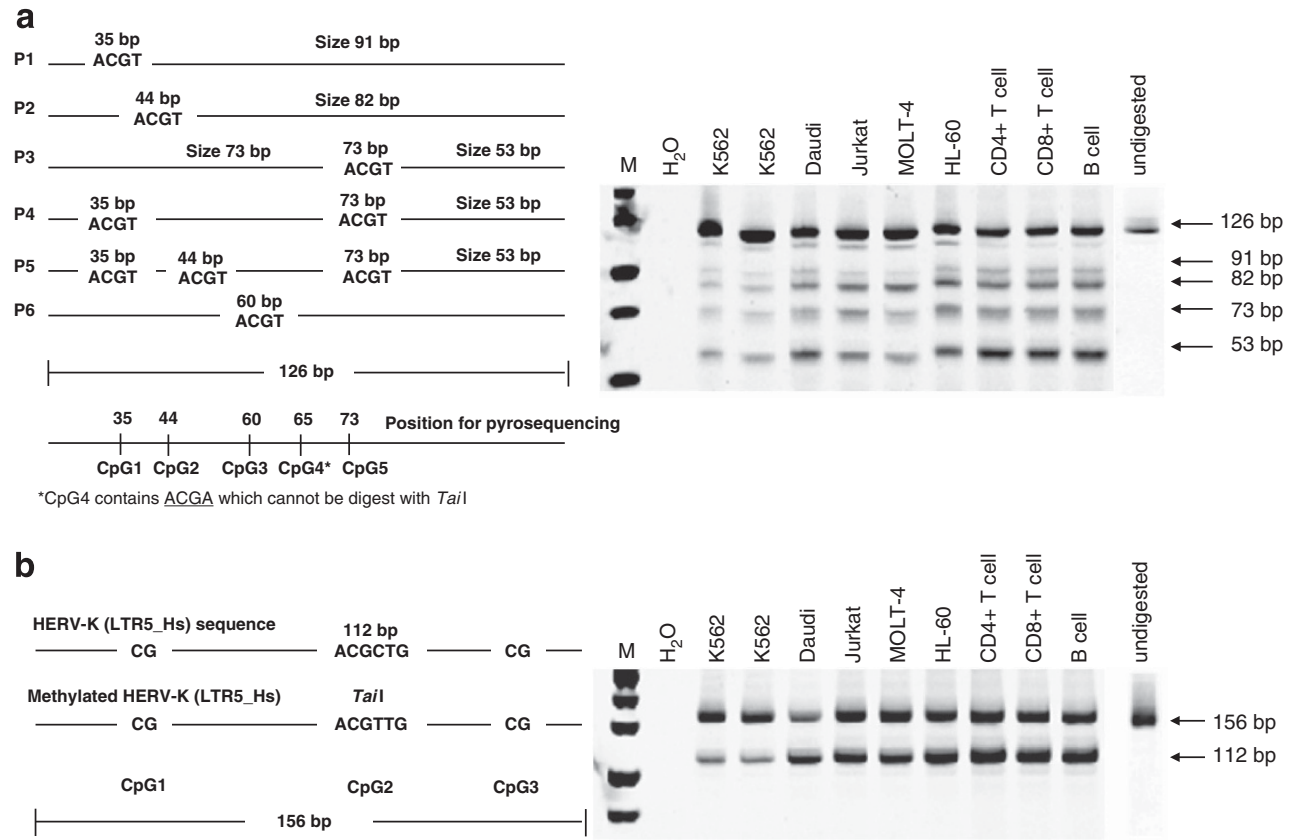

Figure 1 COBRA for HERV-E LTR2C and HERV-K LTR5 Hs. (a) LTR2C and (b) LTR5 Hs amplicon sizes are 126 and 156 bp, respectively. After the DNA was treated with sodium bisulfite, it was later amplified. The PCR products were later digested with Tail. LTR2C methylated sequences yielded 91-, 82-, 73- and 53-bp fragments whereas LTR5_Hs methylated yielded 112- and 44-bp (not detected) fragments. The methylation level was calculated as a percentage of the intensity of the digested methylated fragment divided by the sum of the undigested and digested amplicons. $\mathrm{M}$ represents the quantitative DNA ladder or DNA marker used to assess the size of the bands. Water $\left(\mathrm{H}_{2} \mathrm{O}\right)$ was used as negative control. CpG1-5 (HERV-E) and CpG1-3 (HERV-K) positions according to pyrosequencing method were also indicated in the diagram. 
respectively. As mentioned earlier, we select HERV-E LTR2C and HERV-K LTR5_Hs because previous study reported that PBMCs from SLE and rheumatoid arthritis patients have increased expressions of HERV-E 4-1 and HERV-K10 gag genes, respectively. ${ }^{28,29}$ A total of 265 LTR2C and 618 LTR5_Hs were mapped in the human genome by using Table Browser of UCSC Browser. ${ }^{39}$ We try to develop the primers, which corresponds to conserved regions of all HERV-E LTR2C and HERV-K LTR5_Hs sequence (Figure 1). In addition, our primers allowed the amplification of $\mathrm{CpG}$-containing region that located at $\mathrm{R}$ region of LTR sequence. This region usually has a polyadenylation signal. Furthermore, $\mathrm{R}$ region is important for transcription initiation and regulation of gene expression in retroviruses as described by Benachenhou et al. ${ }^{40}$

To predict how many HERV-E LTR2C and HERV-K LTR5_Hs within the genome that our primers can amplified in bisulfite-treated genomic DNA, we performed an in silico PCR of BiSearch. ${ }^{37,38}$ A total of 77 HERV-E LTR2C $(77 / 265=29 \%)$ and 283 HERV-K LTR5_Hs $(283 / 618=45 \%)$ should be amplified after bisulfite treatment by our primers. The pattern of digestion of 77 HERV-E LTR2C and 283 HERV-K LTR5_Hs PCR products by TaiI was predicted (Supplementary Figures 1 and 2). From the LTR2C-COBRA, multiple bands were detected because there were several restriction sites in the amplicons. ${ }^{41,42}$ Low levels of sequence divergence (1-2\%) in HERV-K sequence were reported. ${ }^{42,43}$ Out of 77 copies of HERV-E LTR2C PCR product, FIVE patterns of restriction site were predicted. We named each pattern as P1-P5 (Figure 1a). According to the sequences, there should be $13 \%$ of $\mathrm{P} 1,15.58 \%$ of $\mathrm{P} 2,14.28 \%$ of $\mathrm{P} 3,27.27 \%$ of $\mathrm{P} 4$ and $28.58 \%$ of P5 (Figure 1a). Only one pattern of HERV-K LTR_Hs was observed (Figure 1b). According to the prediction, there were 1.3 and $10.77 \%$ undigested copies that caused by mutation at Tail regonition site in HERV-E LTR2C of HERV-K LTR5_Hs, respectively. However, it should be noted that the prediction from in silico PCR could not accurately represent the real product as it did not take into account the limitation that can occur in the PCR tube. There are possible cross reaction with similar sequences in the genome as well, so our primer set might be able to amplify more copies than what we have estimated.

Our COBRA was validated using bisulphite-treated fully methylated and unmethylated human control DNA that was mixed in different ratio (Supplementary Figure 3). The result from our COBRA method showed the highly significant positive correlation between observed methylation and actual percentage of methylation. However, we observed a much higher ratio of undigested band than what we have expected. As stated earlier, in silico PCR cannot accurately predict the real experiment. In addition, there might be some degree of undigested copies that was caused by the mutated CpG site from population polymorphism. The percent of coefficients of variance $(\% \mathrm{CV})$ in each group that have significant methylation level, as a measurement for group heterogeneity, were ranging between 2.66$16.31 \%$ (Supplementary Table 1). We also notice some PCR bias especially at the ratios of methylation percentage of 12.5 and $87.5 \%$. Therefore, it should be emphasized that the HERV global methylation in this study is merely an estimation process and cannot display absolute methylation levels. The HERV methylation levels are relative numbers and the levels between samples can be compared only by the same detection protocol.

To further assess the reproducibility of each primer pair, we analyzed the methylated levels of HERV-LTRs in triplicate by using different lymphocyte subsets and cancer cell lines. All cell lines were cultured in at least three flasks so the same cells could be used for the COBRA assay. There were limited deviations in our results (Figure 2).
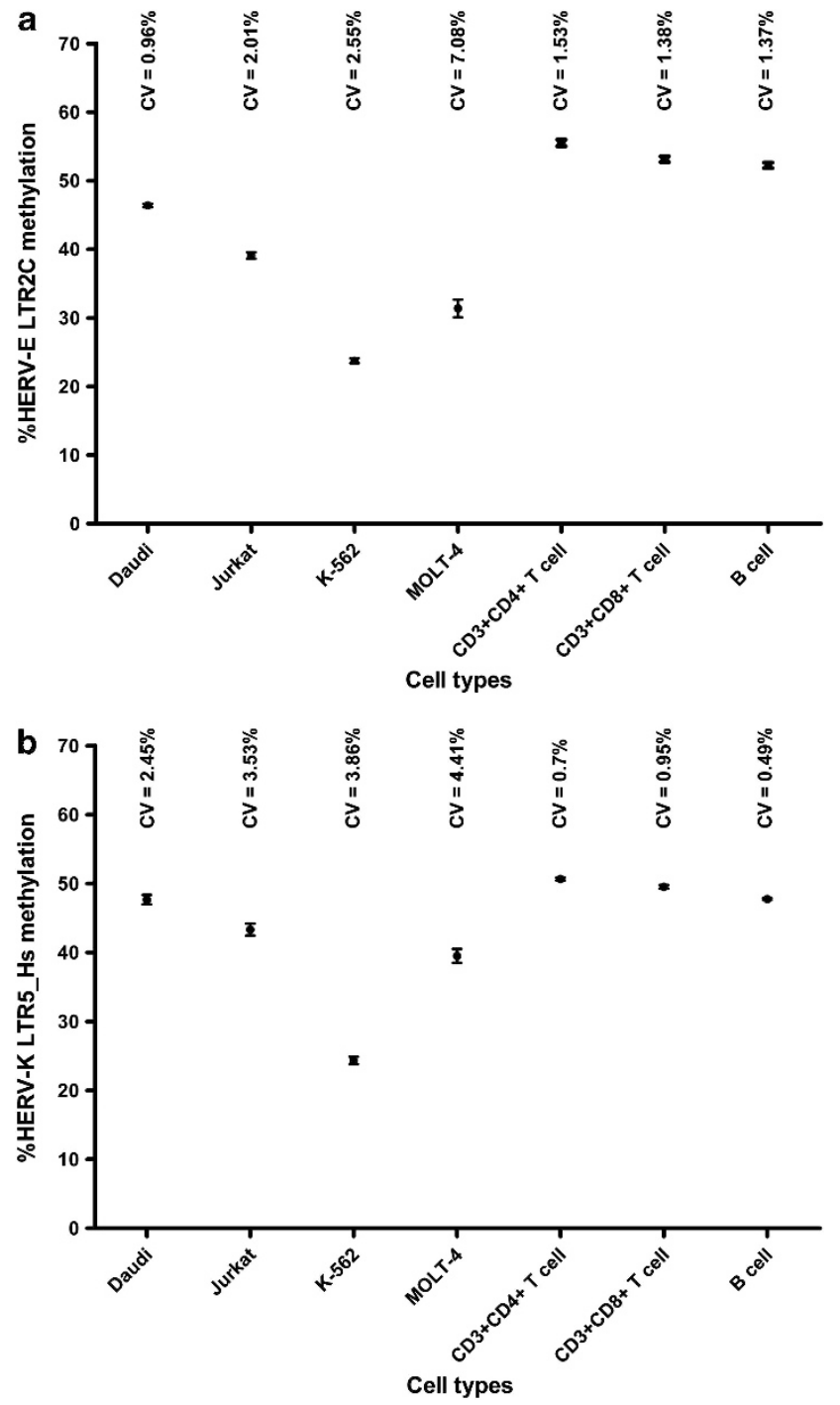

Figure 2 Mean \pm s.e.m. methylated levels of (a) HERV-E LTR2C and (b) HERV-K LTR5_Hs in different cell subtypes. The coefficients of variance $(\% \mathrm{CV})$ were from triplicates for each cell type.

The correlation between COBRA-LTR methylated levels and the mean methylated levels of all CpG by pyrosequencing

In addition, we compared results from the COBRA method to the pyrosequencing technique. Pyrosequencing analysis was performed in 48 samples that were selected by sampling from lymphocyte subsets of 5 normal controls and 10 SLE patients and 3 control cell lines. Methylation levels of five CpG sites of HERV-E and three CpG sites of HERV-K were measured by pyrosequencing. The CpG position analyzed by pyrosequencing was indicated in Figure 1. In general, the methylated levels of each CpG nucleotides, including COBRA TaiI representative nucleotides of HERV-E LTR2C and HERV-K LTR5_Hs, were positively correlated with the mean methylated levels. HERV-E LTR2C was also positively correlated with each CpG dinucleotides $\left(r=0.92, P=1.56 \times 10^{-19}\right.$ for CpG1, $r=0.77, P=1.48 \times 10^{-10}$ for CpG2, $r=0.56, P=3.5 \times 10^{-5}$ for CpG3, $r=0.55, P=6.7 \times 10^{-5}$ for CpG4 and $r=0.49, P=4.1 \times 10^{-4}$ for CpG5). CpG1, CpG2, CpG3 and CpG5 are TaiI CpG sites for COBRA HERV-E LTR2C. Similarly, HERV-K LTR5_Hs was also positively correlated with each CpG dinucleotides $\left(r=0.79, P=1.57 \times 10^{-11}\right.$ for $\mathrm{CpG} 1, r=0.67$, 
$P=2.06 \times 10^{-7}$ for CpG2 and $r=0.64, P=1.07 \times 10^{-6}$ for CpG3, which is TaiI CpG site for COBRA HERV-K LTR5_Hs). Moreover, the correlations between the mean methylated levels from pyrosequencing
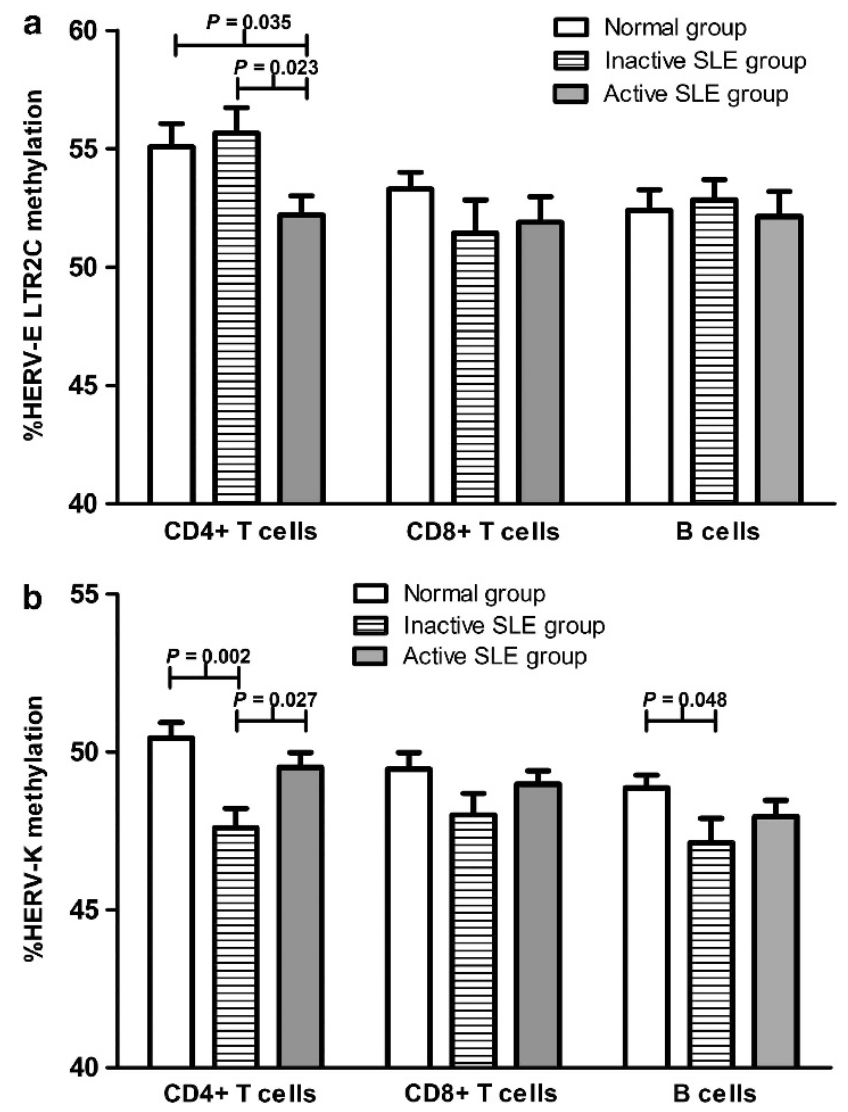

Figure 3 DNA Methylated levels according to the SLE disease activity. Methylated levels of (a) HERV-E LTR2C (b) HERV-K LTR5_Hs were shown in mean \pm s.e.m. $P$-value calculated by unpaired $t$-test two-tailed analysis.
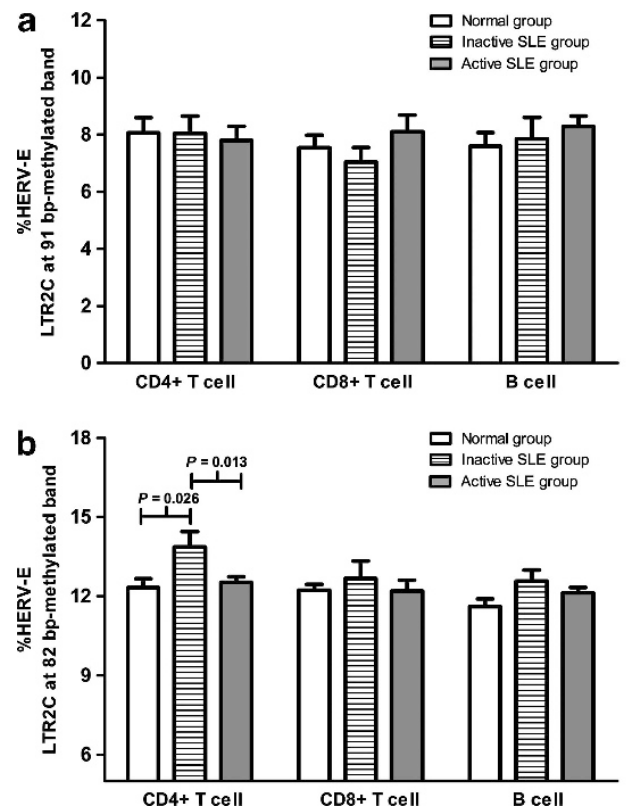

was positively correlated with the COBRA HERV-E LTR2C $(r=0.45$, $P=0.0015)$ and HERV-K LTR5-Hs $(r=0.45, \quad P=0.0003)$. Most importantly, for the CpG2 which is the same as the Tail COBRA HERV-K LTR5-Hs site, there was a highly significant correlation between \% CpG2 methylation calculated by pyrosequencing and \% COBRA HERV-K LTR5_Hs methylation $(r=0.71$, $\left.P=1.38 \times 10^{-8}\right)($ Supplementary Table 2$)$.

Changes in HERV-E LTR2C and HERV-K LTR5_Hs methylation levels in SLE patients according to the activity of the disease Methylated levels in lymphocyte subsets from patients with inactive and active SLE, as well as the controls, were compared with each other. SLE patients were divided into active and inactive groups based on their SLEDAI-2K scores. Methylation of HERV-E LTR2C in $\mathrm{CD} 3+\mathrm{CD} 4+\mathrm{T}$ lymphocytes from patients with active SLE were hypomethylated ( $P=0.023$ and 0.035 , respectively) when compared with inactive and normal controls (Figure 3a). On the other hand, methylation of HERV-K LTR5_Hs in CD3 + CD4 + T lymphocytes from inactive SLE were demethylated $(P=0.002$ and 0.027 , respectively) when compared with normal and active groups. Moreover, demethylation of HERV-K LTR5_Hs in B cells was also detected when compared with the normal controls $(P=0.048)$ (Figure $3 \mathrm{~b})$.

Each band of COBRA HERV-E LTR2C indicated that there were different methylated levels in the PCR products (Figure 4). Therefore, each band of HERV-E LTR2C methylation from SLE patients was compared to the normal controls. Interestingly, hypomethylation in $\mathrm{CD} 3+\mathrm{CD} 4+\mathrm{T}$ lymphocytes and $\mathrm{CD} 3+\mathrm{CD} 8+\mathrm{T}$ lymphocytes were detected at the 53-bp methylated band (band 5) in the patients with active SLE and the normal controls $(P=0.01$ and 0.038 , respectively) (Figure $4 \mathrm{~d}$ ). When the methylated levels in the B cells were compared between the patients with active SLE and the normal controls, we detected a decreasing trend of hypomethylation but this was not significant $(P=0.19)$ (Figure $4 \mathrm{~d})$. Hypermethylation at 82-bp methylated band (band 2) was detected in CD3 + CD4 + T lymphocytes from patients with inactive SLE when compared with the normal controls and the active group $(P=0.026$ and 0.013 ,
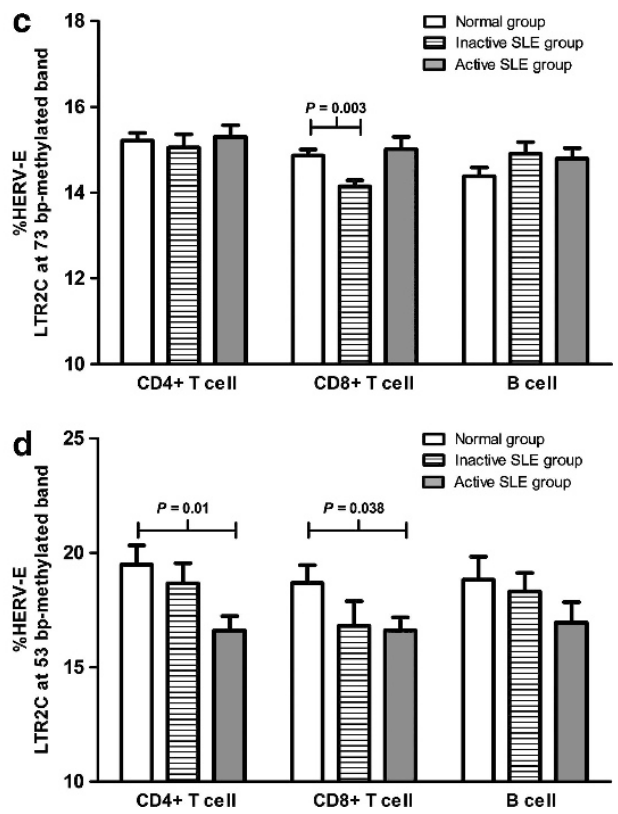

Figure 4 DNA Methylated levels of HERV-E LTR2C in each band. Methylated levels of (a) 91 bp, (b) 82 bp, (c) 73 bp and (d) 53 bp of HERV-E LTR2C in normal controls and SLE lymphocyte subsets were shown in mean \pm s.e.m. $P$-value calculated by unpaired $t$-test two-tailed analysis. 
respectively) (Figure 4b). Furthermore, hypomethylation was also detected at 73-bp methylated band (band 4) in CD3 + CD8 + $\mathrm{T}$ lymphocytes from patients with inactive SLE when compared with the normal controls $(P=0.003)$ (Figure 4c). Therefore, the methylated status of HERV-E LTR2C appears to be different according to their location in the gene.

DNA methylation of LTR2C loci specific

In addition, we compared LTR2C methylation levels of a specific locus between normal and SLE CD4 + T lymphocytes, CD8 $+\mathrm{T}$ and $\mathrm{B}$ lymphocytes by using COBRA for unique HERV-E LTR2C sequence. HERV-E LTR2C-APC2 is located at chromosome $19 \sim 10 \mathrm{~kb}$ at $5^{\prime}$ of adenomatous polyposis coli 2 (APC2) gene. Interestingly, the decrease of methylation levels in CD3 $+\mathrm{CD} 4+\mathrm{T}$ cells and CD3 $+\mathrm{CD} 8+\mathrm{T}$ cells of active SLE patients were observed, although there were no significant difference when compared with normal group $(P=0.053$ and 0.062 , respectively) (Figure 5a). In addition, bisulfite-treated PCR products from normal and active SLE patient were amplified, cloned, and 10 fragments per sample were sequenced. The result from only one pair of normal and active SLE patient shows that percent methylation was lower in active SLE patient compared with healthy individual at this particular locus (Figure 5b).

Association of HERV-E LTR2C and HERV-K LTR5_Hs methylation with clinical parameters

We also correlated the methylated levels of retroelements with the SLEDAI score and clinical parameters such as white blood counts, $\%$ lymphocytes, platelet counts, anti-dsDNA and antinuclear antibodies titer, complement activity ( $\mathrm{CH} 50)$ and quantitative complement levels (C3) (Table 2). Methylated levels of HERV-E LTR2C in $\mathrm{CD} 3+\mathrm{CD} 4+\mathrm{T}$ lymphocytes were positively correlated with leukopenia $(P=0.016, r=0.51)$ and lymphopenia $(P=0.023, r=0.48)$ (Figure $6 \mathrm{a}$ and Table 2). Methylated levels of 82 and 53 methylated band of COBRA HERV-E as well as the LTR2C-APC2 also show the
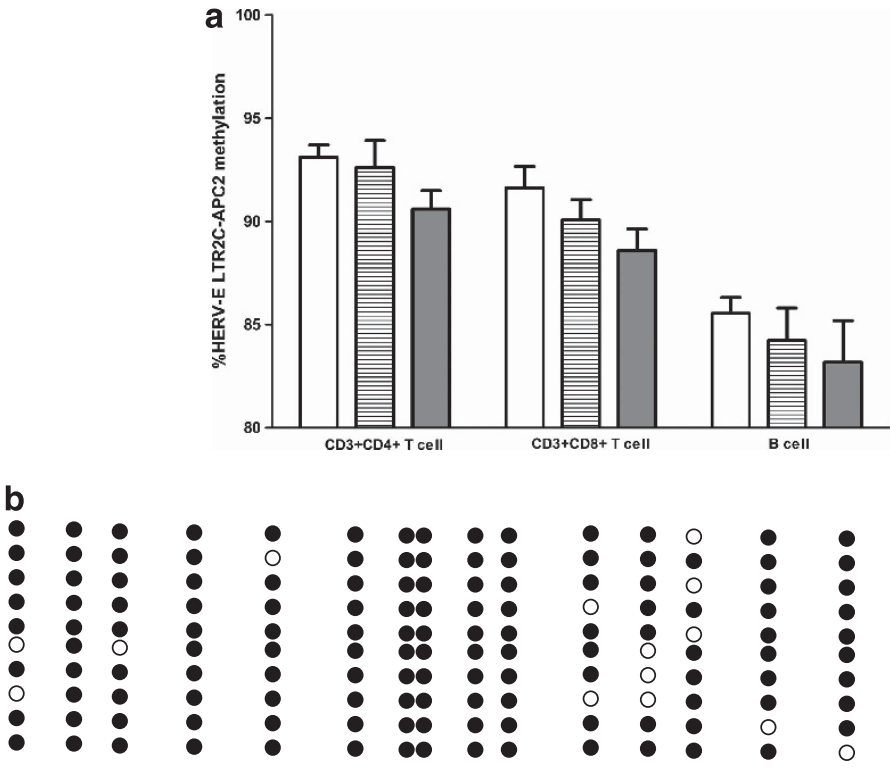

Normal CD3+CD4+ T cell (96\% methylation)
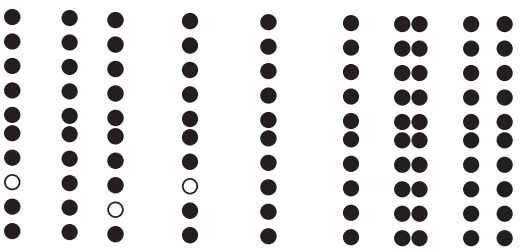

Normal CD3+CD8+ T cell (97\% methylation)

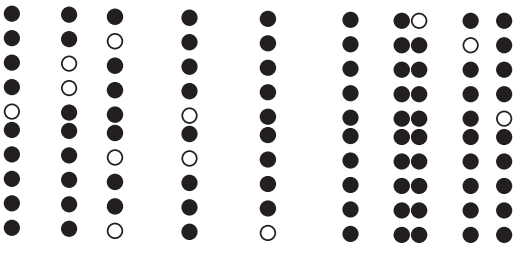

Normal B cell (88\% methylation)

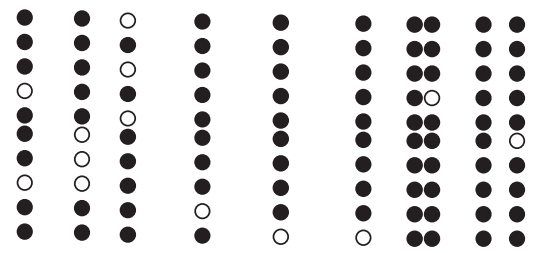

Active SLE CD3+CD4+ T cell ( $87 \%$ methylation)

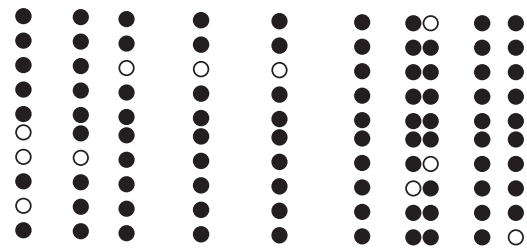

Active SLE CD3+CD8+ T cell (89\% methylation)

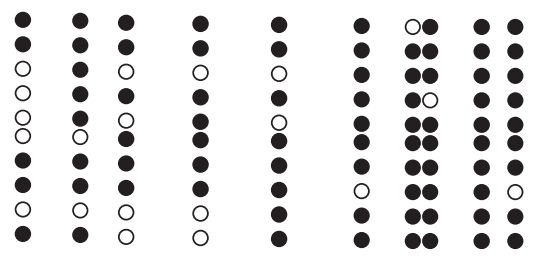

Active SLE B cell ( $80 \%$ methylation)

Figure 5 HERV-E LTR2C APC2 methylation levels. Methylation levels of HERV-E LTR2C APC2 in normal and SLE lymphocyte subsets were shown in mean \pm s.e.m. (a) Bisulfite-treated DNA from normal and active SLE were amplified and cloned, and 10 fragments per donor were sequenced. (b) Black circles correspond to methylated $\mathrm{CpGs}$ and white circles to unmethylated CpGs. P-value calculated by unpaired $t$-test two-tailed analysis. 


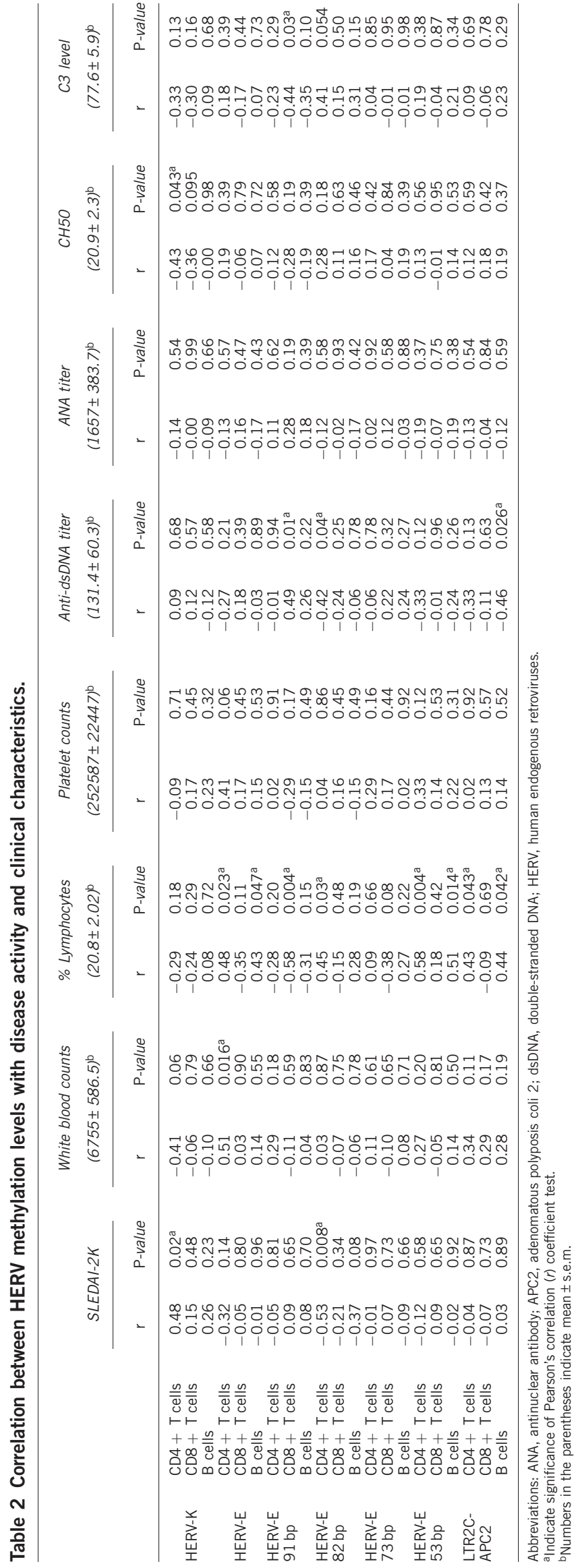

same trend of association with lymphopenia (Table 2). LTR2C HERVK LTR5_Hs in CD3 + CD4 + T lymphocytes was positively correlated with the SLEDAI score $(P=0.02, r=0.48)$ whereas HERV-E LTR2C band 3 was negatively correlated $(P=0.008, r=-0.53$ ) (Figure $6 \mathrm{~b}$ and Table 2). The hypomethylation of HERV-K LTR5_Hs in CD3 + $\mathrm{CD} 4+\mathrm{T}$ lymphocytes was negatively correlated with the complement activity $(P=0.043, r=-0.43)$ (Figure $6 \mathrm{~b}$ ). In addition, methylated levels of HERV-E LTR2C band 2 (91 methylated band) in CD3 + $\mathrm{CD} 8+\mathrm{T}$ lymphocytes with anti-dsDNA titer was positively correlated with the C3 level $(P=0.01, r=0.49)$ (Table 2). Therefore, potential prognostic markers for detecting the activity of SLE disease are the hypomethylated forms of HERV-E and HERV-K. Additional studies are required to determine the function of these hypomethylated forms and its association with the clinical outcome.

\section{DISCUSSION}

Many studies have suggested the potential role of IRSs in regulating cellular functions. As for SLE, it has been shown that hypomethylation of IRSs can affect the cellular gene expression and may contribute to the pathogenesis and activity of the disease. ${ }^{26,44,45}$ In this study, the investigations of hypomethylated HERV's involvement in SLE were carried out for the first time. We have developed the COBRA method to examine the methylated status of HERV-E LTR2C and HERV-K LTR5_Hs. COBRA-IRS was selected because it could detect thousands of $\mathrm{CpG}$ methylated locus just by using one set of conserved primers for each IRS and can be easily used as a screening method. However, the level of global HERV methylation in this study is a rough estimation and cannot display absolute levels.

We detected hypomethylated HERV-E LTR2C but not HERV-K LTR5_Hs in CD3 + CD4 + T lymphocytes of patients with active SLE. This methylation profile also correlates with the fact that HERV-E but not HERV-K transcripts was detected in PBMC of active SLE, supporting a hypothesis that hypomethylation of HERV lead to upregulation of its transcript. ${ }^{28}$ Surprisingly, our result also showed that the patients with inactive SLE had hypomethylation of HERV-K LTR5_Hs in CD3 + CD4 + T and B lymphocytes when compared with the normal control. We do not know the reason of the hypomethylation of HERV-K in the inactive SLE. One hypothesis might be due to the specific function of certain gene regulated by HERV-K as protective factor instead of aggravating factor for disease severity. However, this is purely speculation and such gene is yet to be discovered. The expression of HERV-K transcripts from $\mathrm{CD} 3+\mathrm{CD} 4+\mathrm{T}$ and $\mathrm{B}$ lymphocytes should also be further explored.

Aside from this, there were no correlation between the DNA methylation levels of HERV-E LTR2C and HERV-K LTR5_Hs. It is possible that there are defect in certain proteins that target demethylation process to specific HERV sequences, which remain to be further proved. Currently, there are two main hypotheses regarding the role of HERV in SLE pathogenesis. First, the hypomethylation of HERV might lead to upregulation of HERV transcript. The aberrant expression of HERV could lead to production of autoantibody. ${ }^{46}$ For example, there was a report proposing a cross reaction between (1) HRES-1 p30 gag and the SLE autoantigen U1-snRNP and (2) retroviral Ag p24 and the ribonucleoprotein Sm leading to the production of anti-U1 and anti-Sm antibodies, respectively. ${ }^{29}$ Second, HERV may regulate neighboring genes by acting as promoter, enhancer or altering gene isoform. ${ }^{44}$ The famous example is the report that demethylation in HERV-E alternative promoter of CD5 gene in SLE patients have been shown to upregulate CD5-E1B isoform in SLE B cells. ${ }^{31}$ Therefore, it is possible that these 
a

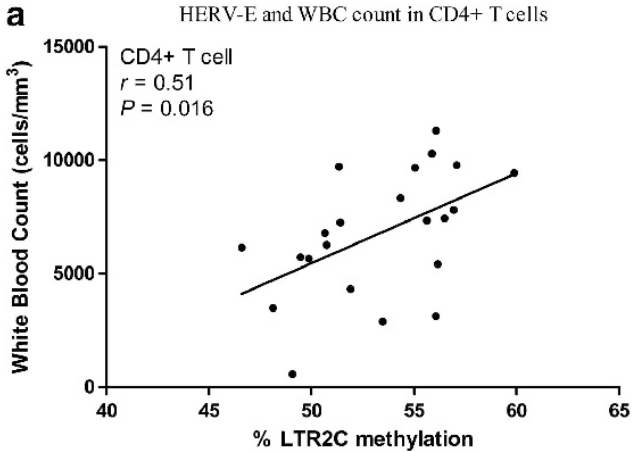

b

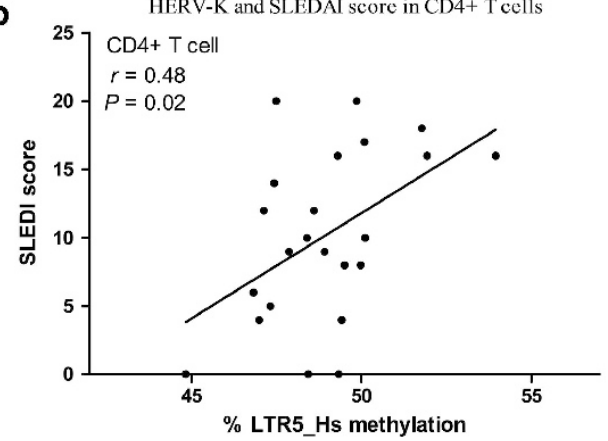

HERV-E and \% lymphocytes in CD4+ T cells

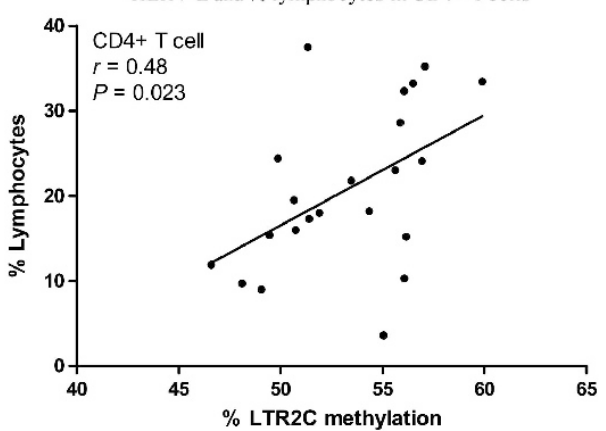

HERV-K and CH50 in CD4+ T cells

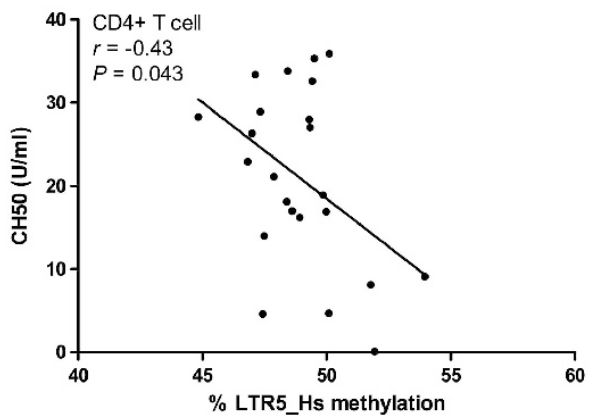

Figure 6 Correlation between DNA methylated levels with disease activity and clinical characteristics. The relationships of HERV-E LTR2C (a) and HERV-K LTR5_Hs (b) methylation levels in lymphocyte subsets to the clinical data and SLEDAI score from SLE patients were shown. Each dot represents an individual patient. P-value indicated significance by Pearson's correlation coefficient test.

hypomethylated HERV-E and HERV-K related to novel genes involved in the pathogenesis of SLE, which remains to be further explored.

To link HERV hypomethylation profile to disease pathogenesis, we analyzed the correlation between the methylated levels and various clinical parameters. Interestingly, hypomethylation of HERV-E LTR2C was positively correlated with leukopenia and lymphopenia in patients with active SLE. Although we cannot assess the association between HERV-E 4-1 with anti-U1 ribonucleoprotein and anti-Sm due to lack of these autoantibodies' result in our patients, the association of HERV-E LTR2C hypomethylation with leukopenia is very much interesting. One recent study also reported that leukopenia is linked to the methylation level.Wang GS et al. ${ }^{47}$ found that the methylation levels in SLE patients with leukopenia were significantly lower than those without leukopenia following UVB irradiation. It is possible that the expression of the IRS antigens on the leukocyte surface can induce the body to produce autoantibodies against the leukocytes or can result in the upregulation of certain genes responsible for this phenomenon. Further studies are needed to confirm and explain these observations.

\section{ACKNOWLEDGEMENTS}

This study was supported by the 90th anniversary of Chulalongkorn University Fund (Ratchadaphiseksomphot Endowment Fund), The Higher Education Research Promotion and National Research University Project of Thailand, Office of the Higher Education Commission (HR1163A), and the Integrated Innovation Academic Center: IIAC, Chulalongkorn University Centenary Academic Development Project (CU56-HR05). JK was supported by the Royal Golden Jubilee PhD Program and Chulalongkorn University. PK was supported by Postdoctoral fellowship from Chulalongkorn University. AM is supported by Research Chair Grant 2011, National Science and Technology
Development Agency, Thailand, and the Four Seasons Hotel Bangkok's 4th Cancer Care charity fun run in coordination with the Thai Red Cross Society and Center of Excellence in Molecular Genetics of Cancer and Human Diseases, Chulalongkorn University. We thank Pirapon June Ohata for editing our paper.

1 Crispin, J. C., Liossis, S. N., Kis-Toth, K., Lieberman, L. A., Kyttaris, V. C., Juang, Y. T. et al. Pathogenesis of human systemic lupus erythematosus: recent advances. Trends Mol. Med. 16, 47-57 (2010).

2 Hedrich, C. M. \& Tsokos, G. C. Epigenetic mechanisms in systemic lupus erythematosus and other autoimmune diseases. Trends Mol. Med. 17, 714-724 (2011).

3 Hughes, T. \& Sawalha, A. H. The role of epigenetic variation in the pathogenesis of systemic lupus erythematosus. Arthritis Res. Ther. 13, 245 (2011).

4 Renaudineau, Y. \& Youinou, P. Epigenetics and autoimmunity, with special emphasis on methylation. Keio J. Med. 60, 10-16 (2011).

5 Richardson, B., Scheinbart, L., Strahler, J., Gross, L., Hanash, S. \& Johnson, M. Evidence for impaired T cell DNA methylation in systemic lupus erythematosus and rheumatoid arthritis. Arthritis Rheum. 33, 1665-1673 (1990).

6 Luo, Y., Li, Y., Su, Y., Yin, H., Hu, N., Wang, S. et al. Abnormal DNA methylation in $\mathrm{T}$ cells from patients with subacute cutaneous lupus erythematosus. $\mathrm{Br}$. J. Dermatol. 159, 827-833 (2008).

7 Balada, E., Ordi-Ros, J., Serrano-Acedo, S., Martinez-Lostao, L., Rosa-Leyva, M. \& Vilardell-Tarres, M. Transcript levels of DNA methyltransferases DNMT1, DNMT3A and DNMT3B in CD4 + T cells from patients with systemic lupus erythematosus. Immunology 124, 339-347 (2008).

8 Balada, E., Ordi-Ros, J., Serrano-Acedo, S., Martinez-Lostao, L. \& Vilardell-Tarres, M. Transcript overexpression of the MBD2 and MBD4 genes in CD4+ $\mathrm{T}$ cells from systemic lupus erythematosus patients. J. Leukoc. Biol. 81, 1609-1616 (2007).

9 Quddus, J., Johnson, K. J., Gavalchin, J., Amento, E. P., Chrisp, C. E., Yung, R. L. et al. Treating activated CD4 + T cells with either of two distinct DNA methyltransferase inhibitors, 5-azacytidine or procainamide, is sufficient to cause a lupus-like disease in syngeneic mice. J. Clin. Invest. 92, 38-53 (1993).

10 Yung, R., Chang, S., Hemati, N., Johnson, K. \& Richardson, B. Mechanisms of drug induced lupus. IV. Comparison of procainamide and hydralazine with analogs in vitro and in vivo. Arthritis Rheum. 40, 1436-1443 (1997).

11 Yung, R., Powers, D., Johnson, K., Amento, E., Carr, D., Laing, T. et al. Mechanisms of drug-induced lupus. II. T cells overexpressing lymphocyte function-associated antigen 
1 become autoreactive and cause a lupus-like disease in syngeneic mice. J. Clin. Invest. 97, 2866-2871 (1996).

12 Kaplan, M. J., Lu, Q., Wu, A., Attwood, J. \& Richardson, B. Demethylation of promoter regulatory elements contributes to perforin overexpression in CD4 + lupus T cells. J. Immunol. 172, 3652-3661 (2004).

$13 \mathrm{Lu}$, Q., Wu, A. \& Richardson, B. C. Demethylation of the same promoter sequence increases CD70 expression in lupus T cells and T cells treated with lupus-inducing drugs. J. Immunol. 174, 6212-6219 (2005).

14 Lu, Q., Wu, A., Tesmer, L., Ray, D., Yousif, N. \& Richardson, B. Demethylation of CD4OLG on the inactive $X$ in T cells from women with lupus. J. Immunol. 179, 6352-6358 (2007).

15 Sugimura, T. \& Ushijima, T. Genetic and epigenetic alterations in carcinogenesis. Mutat. Res. 462, 235-246 (2000).

16 Kaneda, A., Tsukamoto, T., Takamura-Enya, T., Watanabe, N., Kaminishi, M., Sugimura, T. et al. Frequent hypomethylation in multiple promoter $\mathrm{CpG}$ islands is associated with global hypomethylation, but not with frequent promoter hypermethylation. Cancer Sci. 95, 58-64 (2004).

17 Bannert, N. \& Kurth, R. Retroelements and the human genome: new perspectives on an old relation. Proc. Natl Acad. Sci. USA 101 (Suppl 2), 14572-14579 (2004).

18 Levy, S., Sutton, G., Ng, P. C., Feuk, L., Halpern, A. L., Walenz, B. P. et al. The diploid genome sequence of an individual human. PLoS Biol. 5, e254 (2007).

19 Jordan, I. K., Rogozin, I. B., Glazko, G. V. \& Koonin, E. V. Origin of a substantial fraction of human regulatory sequences from transposable elements. Trends Genet. 19, 68-72 (2003).

20 Aporntewan, C., Phokaew, C., Piriyapongsa, J., Ngamphiw, C., Ittiwut, C., Tongsima, S. et al. Hypomethylation of intragenic LINE-1 represses transcription in cancer cells through AGO2. PLoS One 6, e17934 (2011).

21 Cordaux, R. \& Batzer, M. A. The impact of retrotransposons on human genome evolution. Nat. Rev. 10, 691-703 (2009).

22 Kitkumthorn, N. \& Mutirangura, A. Long interspersed nuclear element-1 hypomethylation in cancer: biology and clinical applications. Clin. Epigenetics 2, 315-330 (2011).

23 Saito, K., Kawakami, K., Matsumoto, I., Oda, M., Watanabe, G. \& Minamoto, T. Long interspersed nuclear element 1 hypomethylation is a marker of poor prognosis in stage IA non-small cell lung cancer. Clin. Cancer Res. 16, 2418-2426 (2010).

24 Pattamadilok, J., Huapai, N., Rattanatanyong, P., Vasurattana, A., Triratanachat, S., Tresukosol, D. et al. LINE-1 hypomethylation level as a potential prognostic factor for epithelial ovarian cancer. Int. J. Gynecol. Cancer 18, 711-717 (2008).

25 Iramaneerat, K., Rattanatunyong, P., Khemapech, N., Triratanachat, S. \& Mutirangura, A. HERV-K hypomethylation in ovarian clear cell carcinoma is associated with a poor prognosis and platinum resistance. Int. J. Gynecol. Cancer 21, 51-57 (2011).

26 Nakkuntod, J., Avihingsanon, Y., Mutirangura, A. \& Hirankarn, N. Hypomethylation of LINE-1 but not Alu in lymphocyte subsets of systemic lupus erythematosus patients. Clinica chimica acta; Int. J. Clin. Chem. 412, 1457-1461 (2011).

27 Jintaridth, P. \& Mutirangura, A. Distinctive patterns of age-dependent hypomethylation in interspersed repetitive sequences. Physiol. Genomics 41, 194-200 (2011).

28 Okada, M., Ogasawara, H., Kaneko, H., Hishikawa, T., Sekigawa, I., Hashimoto, H. et al. Role of DNA methylation in transcription of human endogenous retrovirus in the pathogenesis of systemic lupus erythematosus. J. Rheumatol. 29, 1678-1682 (2002).

29 Piotrowski, P. C., Duriagin, S. \& Jagodzinski, P. P. Expression of human endogenous retrovirus clone 4-1 may correlate with blood plasma concentration of anti-U1 RNP and anti-Sm nuclear antibodies. Clin. Rheumatol. 24, 620-624 (2005).
30 Ejtehadi, H. D., Freimanis, G. L., Ali, H. A., Bowman, S., Alavi, A., Axford, J. et al. The potential role of human endogenous retrovirus K10 in the pathogenesis of rheumatoid arthritis: a preliminary study. Ann. Rheum. Dis. 65, 612-616 (2006).

31 Garaud, S., Le Dantec, C., Jousse-Joulin, S., Hanrotel-Saliou, C., Saraux, A., Mageed, R. A. et al. IL-6 modulates CD5 expression in B cells from patients with lupus by regulating DNA methylation. J. Immunol. 182, 5623-5632 (2009).

32 Gladman, D. D., Ibanez, D. \& Urowitz, M. B. Systemic lupus erythematosus disease activity index 2000. J. Rheumatol. 29, 288-291 (2002).

33 Uribe, A. G., Vila, L. M., McGwin, G. Jr, Sanchez, M. L., Reveille, J. D. \& Alarcon, G. S. The Systemic lupus activity measure-revised, the Mexican Systemic Lupus Erythematosus Disease Activity Index (SLEDAI), and a modified SLEDAI-2K are adequate instruments to measure disease activity in systemic lupus erythematosus. J. Rheumatol. 31, 1934-1940 (2004).

34 Moskalev, E. A., Zavgorodnij, M. G., Majorova, S. P., Vorobjev, I. A., Jandaghi, P., Bure, I. V. et al. Correction of PCR-bias in quantitative DNA methylation studies by means of cubic polynomial regression. Nucleic Acids Res. 39, e77 (2011).

35 Miller, S. A., Dykes, D. D. \& Polesky, H. F. A simple salting out procedure for extracting DNA from human nucleated cells. Nucleic Acids Res. 16, 1215 (1988)

36 Jurka, J. Repbase update: a database and an electronic journal of repetitive elements. Trends Genet. 16, 418-420 (2000).

37 Tusnady, G. E., Simon, I., Varadi, A. \& Aranyi, T. BiSearch: primer-design and search tool for PCR on bisulfite-treated genomes. Nucleic Acids Res. 33, e9 (2005).

38 Aranyi, T., Varadi, A., Simon, I. \& Tusnady, G. E. The BiSearch web server. BMC Bioinform. 7, 431 (2006).

39 Kent, W. J., Sugnet, C. W., Furey, T. S., Roskin, K. M., Pringle, T. H., Zahler, A. M. et al. The human genome browser at UCSC. Genome Res. 12, 996-1006 (2002).

40 Benachenhou, F., Jern, P., Oja, M., Sperber, G., Blikstad, V., Somervuo, P. et al. Evolutionary conservation of orthoretroviral long terminal repeats (LTRs) and ab initio detection of single LTRs in genomic data. PLoS One 4, e5179 (2009).

41 Taruscio, D., Floridia, G., Zoraqi, G. K., Mantovani, A. \& Falbo, V. Organization and integration sites in the human genome of endogenous retroviral sequences belonging to HERV-E family. Mamm. Genome 13, 216-222 (2002).

42 Reiss, D., Zhang, Y. \& Mager, D. L. Widely variable endogenous retroviral methylation levels in human placenta. Nucleic Acids Res. 35, 4743-4754 (2007).

43 Belshaw, R., Dawson, A. L., Woolven-Allen, J., Redding, J., Burt, A. \& Tristem, M. Genomewide screening reveals high levels of insertional polymorphism in the human endogenous retrovirus family HERV-K(HML2): implications for present-day activity. J. Virol. 79, 12507-12514 (2005).

44 Balada, E., Ordi-Ros, J. \& Vilardell-Tarres, M. Molecular mechanisms mediated by human endogenous retroviruses (HERVs) in autoimmunity. Rev. Med. Virol. 19, 273-286 (2009).

45 Crow, M. K. Long interspersed nuclear elements (LINE-1): potential triggers of systemic autoimmune disease. Autoimmunity 43, 7-16 (2010).

46 Perl, A., Fernandez, D., Telarico, T. \& Phillips, P. E. Endogenous retroviral pathogenesis in lupus. Curr. Opin. Rheumatol. 22, 483-492 (2010).

47 Wang, G. S., Zhang, M., Li, X. P., Zhang, H., Chen, W., Kan, M. et al. Ultraviolet B exposure of peripheral blood mononuclear cells of patients with systemic lupus erythematosus inhibits DNA methylation. Lupus 18, 1037-1044 (2009).

Supplementary Information accompanies the paper on Journal of Human Genetics website (http://www.nature.com/jhg) 